\title{
Barefoot-Pedestrian Tribometry: In Vivo Method of Measurement of Available Friction between the Human Heel and the Walkway
}

\author{
Marcus BESSER ${ }^{1}$, Mark MARPET ${ }^{2 *}$ and Howard MEDOFF $^{3}$
}

\author{
${ }^{1}$ Thomas Jefferson University, 130 S. Ninth Street, Philadelphia, Pennsylvania 19107, USA \\ ${ }^{2}$ St. John's University, 300 Howard Avenue, Staten Island, New York 10301, USA \\ ${ }^{3}$ Pennsylvania State University, 1600 Woodland Road, Abington, Pennsylvania 19001, USA
}

Received June 26, 2007 and accepted November 7, 2007

\begin{abstract}
We have developed an in vivo tribometer for characterizing the friction of a human heel against a planar test surface. The pedestrian steps down on an angled test surface and an observer determines if the person's heel slips. In the simplest variation, the subject simply steps down onto the test surface. The second variation has the standing subject's lower leg constrained to a vertically running carriage, forcing the pedestrian's leg to descend vertically. The third variation has the subject sitting, with an operator raising and lowering the carriage to which the leg is attached. The test surface was fixed at a given angle, a set of repeated tests was run, and the number of tests and slips were recorded. The test-surface angle was incremented through a range that varied from no slips to all slips. We analyzed the data using logistic regression. We found that the unconstrained test subject's logistic-regression curves varied greatly from subject to subject. The standing, constrained subjects were significantly closer to each other, but at the expense of a much higher spread of the angular range. The seated, constrained test subject's results were both extremely close and had extremely low angular spread.
\end{abstract}

Key words: Skin tribometry, Barefoot friction, Walkway safety, Slip and fall, Friction metrology

\section{Introduction}

While the characterization of the frictional interface between a shod pedestrian and a walkway is the subject of considerable research, the subject of the friction between a test surface and a barefoot-pedestrian has received essentially no attention. In spite of this, the subject is of considerable importance, because swimmingand bathing-facility accidents are capable of producing significant harm. Barefoot-Pedestrian Tribometry (BPT)specific methods today are limited in scope and value, and the use of shoe-bottom test feet will simply not produce meaningful results. Medical Silicone, viz., the testfoot material used in ASTM F 462 Standard Consumer Safety Specification for Slip-Resistant Bathing Facilities is sometimes used as a barefoot-skin surrogate, but its biofidelity has never been explored, let alone established.

*To whom correspondence should be addressed.
Not only are shoe-bottom and medical-silicone test-foot materials completely different from human skin, but there is a friction-ridge pattern existing on the skin surface that evolved to enhance friction. Thus, to be meaningful, either a tribometric surrogate for human skin needs to be developed (or discovered) or a test regimen actually utilizing human skin needs to be worked out. We have chosen the latter path.

\section{Research background-Skin friction}

Naylor ${ }^{1)}$ developed an in vivo instrument to measure dynamic skin friction. He found that human skin under certain conditions followed the classical friction laws. He found that sweat, lubrication, and skin condition had a profound impact on measured friction. Comaish and Bottoms $^{2)}$, using a weight-driven, drag-sled-like skin tribometer, hypothesize that the coefficient of friction is related to the normal force by a more complex relationship than postulated by Amontons, viz., $F \sim \mu \cdot W^{n}$. 
Comaish et al. ${ }^{3)}$ developed a hand-held in vivo tribometer consisting of a rotating-at-constant-velocity, constant-load annular test foot. Cua et al. ${ }^{4)}$ used the tribometer described in Comaish ${ }^{3)}$ to test dynamic skin friction and other parameters on various parts of the bodies of young and old Caucasian males and females without skin disease. (The heelstrike areas were not tested.) The results reportedly varied significantly with respect to the different body areas, but not with respect to age or gender.

Loden et al.5) studied in vivo skin-friction differences between normal and atopic (allergy-related dermatitis) skin with respect to friction and other parameters. Asserin et al. ${ }^{6)}$ describes the development of an in vivo skin tribometer using a ruby indenter, $3 \mathrm{~mm}$ diameter, loaded using light weights, and designed to test the volar forearm.

Egawa et al. ${ }^{7)}$ describes measurements made by sliding a "fingerprint simulator" - a set of 20 piano wires arranged in parallel approximately 0.02 inches apart and arranged perpendicular to the direction of sliding. The studied parameter was the integral of the friction coefficient over one inch of sliding, which did not vary with load over a $0.3 \mathrm{~N}$ load range, or with age.

Sivamani et al ${ }^{8)}$ and Gitis and Sivamani ${ }^{9)}$ tested in vivo the forearms of volunteers classified by age, gender, and ethnicity. Significant differences were not found. Sivamani et $a l .{ }^{10)}$ reviewed past studies of skin tribometry, including summaries of the different test feet and friction-coefficient ranges found.

Li et al. ${ }^{11)}$ describe experiments to characterize the rubbing that occurs between skin and "external surfaces." Tests on a single subject involved reciprocal loading under various force-displacement conditions using a test foot fabricated from a prosthetic-socket material.

In summary, although skin friction has been studied, these studies have either not used living tissue, or have done so without attempting biofidelity. Devices described in these studies often dragged a small weight over the forearm-there is no evidence that such results would apply to the plantar surface of the foot and the larger loads and loading rates involved with walking. Clearly, a different approach to the problem must be found.

Importantly, there is strong evidence that skin friction varies with skin hydration, and no significant evidence that it varies with respect to age, gender, or ethnicity.

\section{Research background-The apparatus}

Researchers have used various tribometric devices to measure barefoot-pedestrian friction. For the most part, surrogate test-foot materials have been utilized in place of human skin.

Brungraber ${ }^{12)}$, in an effort to obtain information about the hazards associated with barefoot walking around swimming pool decks, developed a ramp apparatus to measure the coefficient of friction between a barefooted test subject and representative pool decking materials. The equipment consisted of a variably inclined plane incorporated into a test frame. Test results were compared to those obtained using a Slip-Test Mark I tribometer (the Portable Articulated-Strut Tribometer) on the same pool-deck-material test surfaces.

DIN 5109713) is a German National Standard that utilizes an adjustable-slope ramp with specially-trained, "calibrated" pedestrians walking upon it. It is used to evaluate the safety of candidate floor coverings, including wet-loaded barefoot areas, such as bathing facilities. A continuous and uniform stream of contaminating fluid is applied to the surface of the test floor covering. It is not clear that walking on the ramp using "standardized stepping" is biofidelic with respect to ordinary human ambulation.

RAPRA CH00014) is similar to DIN 51130 protocol, but water, rather than motor oil is used upon the test surfaces as the lubricant. This protocol has the same advantages, and suffers from the same deficits, as DIN 51130.

Medoff et al. ${ }^{15)}$ describes a far simpler step ramp used to evaluate friction of walkway surfaces and/or shoe bottoms. The subject does not walk on the ramp but, rather, drops the foot onto the water-lubricated test surface. (A modified version of the ramp tester described in this paper was used in our experiments.)

\section{Research background-Logistic regression}

Hanson et al. ${ }^{16)}$ used logistic regression to predict falls (falling/not falling was the dichotomous dependent variable); the difference between the required friction and the utilized friction was the dependant variable.

Burnfield and Powers ${ }^{17)}$ set pedestrians across a lubricated force-plate, and used logistic regression to predict pedestrian slips, separately using the difference between available and utilized/required friction (as did Hanson ${ }^{16)}$ ) and just available friction as the independent variable. They determined that, while the friction difference gave the most accurate results, available friction alone also proved "accurate."

Marpet and Medoff ${ }^{18)}$, and Marpet and Medoff ${ }^{19)}$ describe an apparatus that substitutes weights for the friction force at the interface between the test foot and test surface of a binary-output tribometer (a Portable Inclinable Articulated Strut Tribometer (PIAST)). Repeated tests at a given angular setting allow for an estimate of the probability of a slip. Logistic regression was used to model the characteristic function of the tribometer (This paper uses the mathematical model developed in these papers). 
Medoff et al. ${ }^{21)}$ extend the work presented in Marpet and Medoff ${ }^{18)}$ and Marpet and Medoff ${ }^{19)}$ to the comparison of two different binary-output tribometers: the Slip-Test Mark II and the Mark III. To accomplish this, an air-bearing, "zero-friction" test carriage was developed and utilized.

Besser et $a l .{ }^{20)}$, Marpet et al. ${ }^{22)}$, and Medoff et al. ${ }^{23)}$ discuss in detail the comparison of the two related tribometers in Medoff et al. ${ }^{21)}$

\section{Subjects and Methods}

\section{Instrumentation}

As has been stated, a modified version of the ramp tester described by Medoff et al. ${ }^{15}$ ) was used to conduct these experiments. The original device consisted of an adjustable platform onto which a standing test subject could step. The angle of the platform can be adjusted using a hydraulic jack; ramp angle was measured with an SPI-Tronic Pro 3600 electronic level, with $\pm 0.05^{\circ}$ accuracy to $10^{\circ}$, and $\pm 0.2^{\circ}$ from $10^{\circ}-80^{\circ}$. The test surface (onto which the subject steps) can be changed, and was continuously wetted by means of a circulation pump and a water reservoir.

Modifications to the machine were made in order to ensure that the stepping leg would strike the test surface in a repeatable manner, and in a vertical direction. A fiberglass cast was made of the lower leg of each subject; this cast was affixed to a carriage, which was constrained to travel vertically under the influence of sleeve bearings running along vertical rods mounted to the frame of the ramp tester. The cast was pinned to the carriage at the lateral proximal point, just below the tibial plateau of the right knee. A single pin joint was used at this location to allow the leg to pivot forward in the case of a slip.

For the third test variation, an additional modification was made to the machine to allow the subject to be seated during testing. The constrained leg was positioned such that the hip and knee were at approximately $90^{\circ}$ of flexion. For these experiments, one of the experimenters lifted the carriage to which the cast was attached approximately $10 \mathrm{~cm}$ (4"), and released it, allowing the test leg to 'fall' onto the test surface.

For all three test variations, the same test surface of wetted polished marble was used.

For all three test variations, two researchers visually observed the foot/marble contact, each from a distance of approximately $30 \mathrm{~cm}$. The observers focused solely on the determination as to whether a slip did or did not occur. Because the contact between the heel and the angled ramp surface occurred at a repeatable position on the test surface, and because the subject was 'stationary,' the task of determining whether or not a slip had occurred was far less difficult than it would have been if the subject had been ambulating on a walkway. Reinforcing our confidence in the ability of the observers to detect a slip is the fact that there was very little disagreement between the two observers. (In the event that the observers disagreed, that test was not used.)

\section{Subjects}

Two of the researchers (HM, MB) served as test subjects for this study. The subjects are healthy adult men, with no previous medical history of injury to the right foot, specifically the heel.

\section{Procedure}

Before testing, subjects had their lower right legs cast by a casting technician in the orthopedics department of a large Academic Medical Center hospital. Standard fiberglass cast techniques were used. The cast reached from just proximal to the medial and lateral maleoli of the ankle to just below the tibial plateau of the knee; the cast did not interfere with either knee or ankle motion. The lateral side of the cast was extended slightly to allow for attachment to the testing machine. The cast was cut down the front to allow it to be donned and doffed by the test subject.

For all tests, subjects were in bare feet. No special preparation of the foot or heel was done. Cursory inspection of the feet revealed no unusual anatomic abnormalities or skin conditions.

Three different tests were conducted on each of the subjects. For all of the tests, the test surface was constantly wetted using a recirculating pump. The subject's foot was allowed to 'hydrate' for ten minutes before testing began, with the test surface horizontal (ramp angle $=0^{\circ}$ ). For each test, the ramp angle was increased in increments until the subject slipped, and then lowered slightly and testing began. At each ramp angle, the subject would step onto the ramped surface repeatedly but slowly (typically 20 repeats at each setting). For each step, researchers would observe the foot strike, and determine whether a slip had occurred. The probability of slipping at a given angle was calculated as follows:

$$
p(\text { slip })=\frac{(\# \text { of slips detected })}{(\# \text { of steps onto ramp })}
$$

Data were collected for angles ranging from those where no slips were detected $[p(\operatorname{slip})=0.0]$ until reaching an angle where slip was detected for all steps $[p($ slip $)=1.0]$. Ramp angle was increased in approximately $0.1^{\circ}-0.2^{\circ}$ increments.

\section{Test variation 1: Unconstrained standing}

Subject was positioned standing in the ramp tester, with 
the left foot supporting their weight on a horizontal platform and the right foot unsupported over the test surface. The subject was unconstrained-that is, the leg was free to move in all directions.

Test variation 2: Constrained and standing (See Fig. 1)

Subject was again positioned standing in the ramp tester, supporting their weight on a horizontal platform and the right foot unsupported over the test surface. For these trials, the right calf was constrained by the fiberglass cast previously described. The cast was secured to the leg by closing the cast seam with Velcro. The proximal lateral aspect of the cast was pinned to the carriage which was free to travel vertically (linear bearings assured unimpeded motion), but which prevented horizontal translation of the leg. This forced the lower leg to travel in a vertical direction when striking the test surface.

Test variation 3: Constrained and seated (See Fig. 2)

A seat was added to the ramp tester which would allow the subject to sit with their hip and knee angles at approximately $90^{\circ}$. The test leg was once again constrained in the cast, and pinned to the vertical carriage as described above. For the seated trials, the test subject did not initiate the movement to step onto the test surface; instead, a researcher would lift the carriage approximately $10 \mathrm{~cm}$ (4") and 'drop' the foot onto the test surface.

\section{Mathematical background_Logistic regression}

Ordinary regression: fitting a line (or curve) to a dataset, is commonly taught in elementary-statistics courses. When the dependant variable can take on only two values, Bernoulli distributed, then logistic regression must be used. An elementary reference for the subject may be found at wikipedia ${ }^{24)}$

A Bernoulli variable is one that can take on the values 0 or 1 (equivalent to True or False, Success or Failure) such that:

$$
\begin{aligned}
& p(0)=p \geq 0 \\
& p(1)=(1-p) \geq 0 \\
& p(0)+p(1)=1
\end{aligned}
$$

Logistic regression is based upon the logit function (the logarithm of the odds ratio):

$$
\operatorname{logit}(p)=\ln \left(\frac{p}{1-p}\right)
$$

In logistic regression, the dependant variable is a logit, and is set to the predictor (here, we use the simple-linear-regression predictor, $y_{i}=\beta_{0}+\beta_{1} x$ ):

$$
\operatorname{logit}\left(p_{i}\right)=\ln \left(\frac{p_{i}}{1-p_{i}}\right)=\beta_{0}+\beta_{1} x_{i}
$$

The solution to the model is:

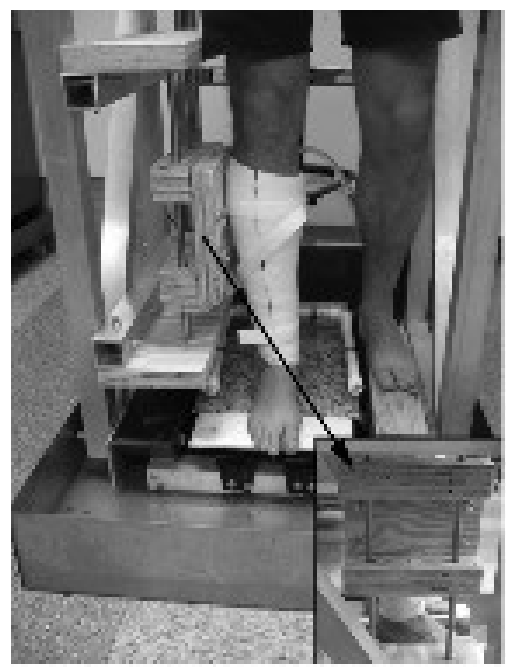

Fig. 1. Subject's leg vertically constrained in ramp apparatus (_CV).

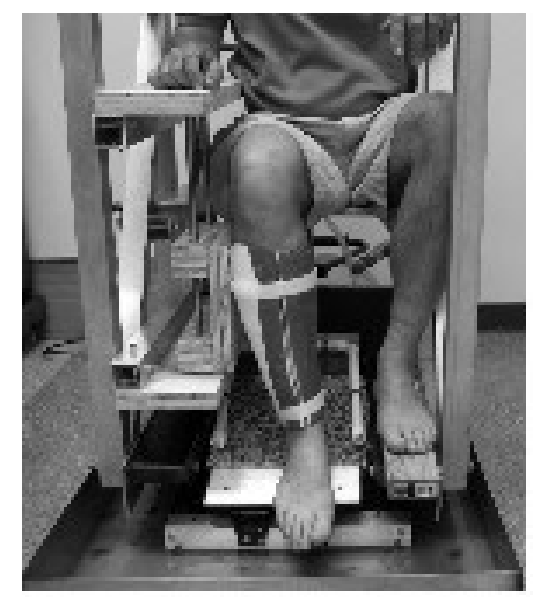

Fig. 2. Sitting subject with lower-leg vertically constrained (_CS).

$$
p_{i}=\mathrm{P}\left(y_{i}=0 \mid x_{i}\right)=1-\frac{1}{1+e^{-\left(\beta_{0}+\beta_{1} x_{i}\right)}}
$$

The effect of varying the parameters is analogous to that in linear regression; varying $\beta_{0}$, the y-intercept in linear regression, in effect shifts the curve to the left or right, as seen in Fig. 3. ( $\beta_{1}$ is held constant.)

Varying $\beta_{1}$, the slope in simple linear regression, has the effect of 'squaring the corners' of the s-curve. (See Fig. 4. Here, $\beta_{0}$ is held constant.)

The way a logistics-regression curve is interpreted is similar to the way any regression is interpreted, save the fact that the dependent variable is a probability. For example, using the $\beta_{0}=35$ curve in the Fig. 1 , we see that with the test ramp set at a $2.5^{\circ}$ angle, there is about a $25 \%$ chance of a person slipping during any test. In a series of 20 tests, a typical test run, we would expect to 


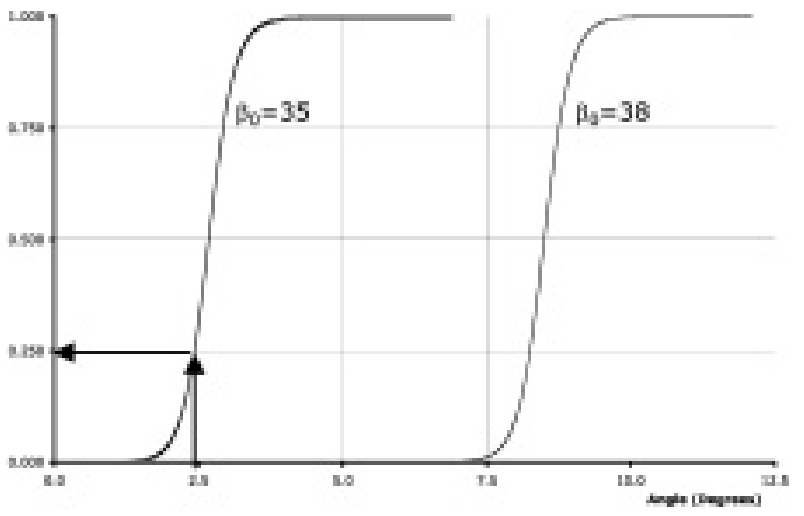

Fig. 3. The effect of changing $\beta_{0}\left(\beta_{1}\right.$ constant).

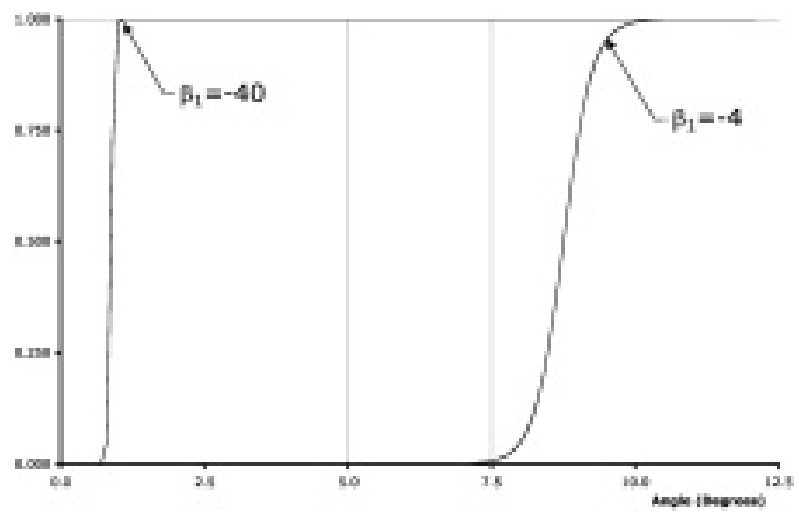

Fig. 4. The effect of changing $\beta_{1}$ ( $\beta_{0}$ constant).

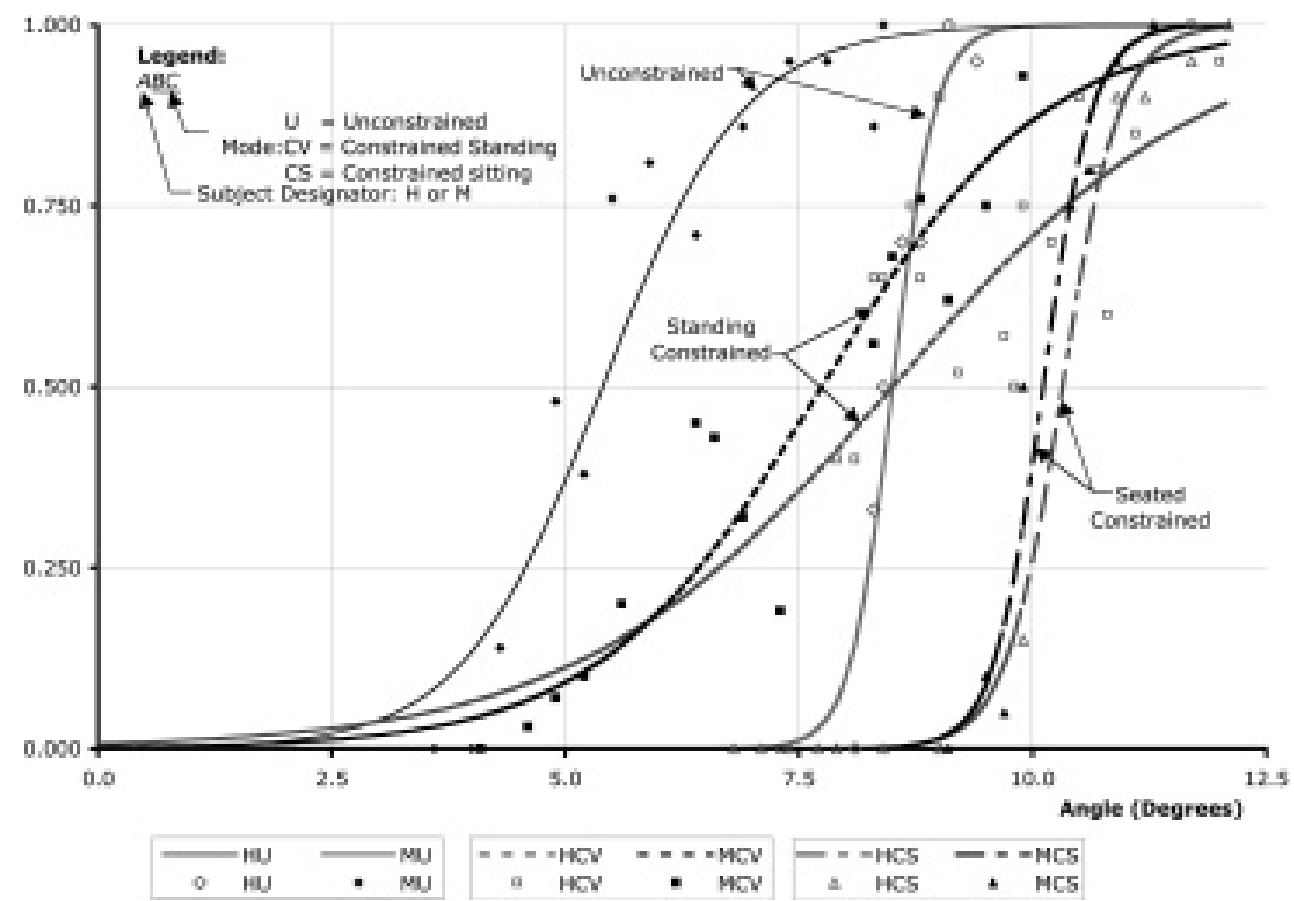

Fig. 5. The Logistic Regression Results.

see five slips.

\section{Results}

For each test, for each subject, a logistic regression was calculated to fit the data using JMP, a commonly used statistical-analysis and experimental design program. The dependent variable was whether a slip occurred $\left(y_{i}=1\right)$ or did not occur $\left(y_{i}=0\right)$; the dependant variable was the ramp angle, in degrees. Graphs of these logistic-regression predictor equations, as well as the summary-data scatter (the probability of a slip at a given angle) are shown in Fig. 5. In this figure, data are plotted for each subject $(\mathrm{H}, \mathrm{M})$, for each condition $(\mathrm{U}=$ unconstrained, $\mathrm{CV}=$ constrained standing (or vertical), CS = constrained seated), for a total
Table 1. Regression-equation parameter estimates

\begin{tabular}{lccc}
\hline Condition & Subject & Intercept $\left(\beta_{\mathbf{0}}\right)$ & Slope $\left(\beta_{\mathbf{1}}\right)$ \\
\hline Unconstrained & $\mathrm{H}$ & 37.78 & -4.44 \\
& $\mathrm{M}$ & 7.21 & -1.34 \\
Constrained Standing & $\mathrm{H}$ & 5.02 & -0.59 \\
& $\mathrm{M}$ & 6.48 & -0.84 \\
Constrained Seated & $\mathrm{H}$ & 33.73 & -3.27 \\
& $\mathrm{M}$ & 38.68 & -3.83 \\
\hline
\end{tabular}

of six curves. The parameter estimates for the intercepts and slopes used to calculate the graphs are shown in Table 1. All parameter estimates were highly significant $(p<0.0001)$ : 


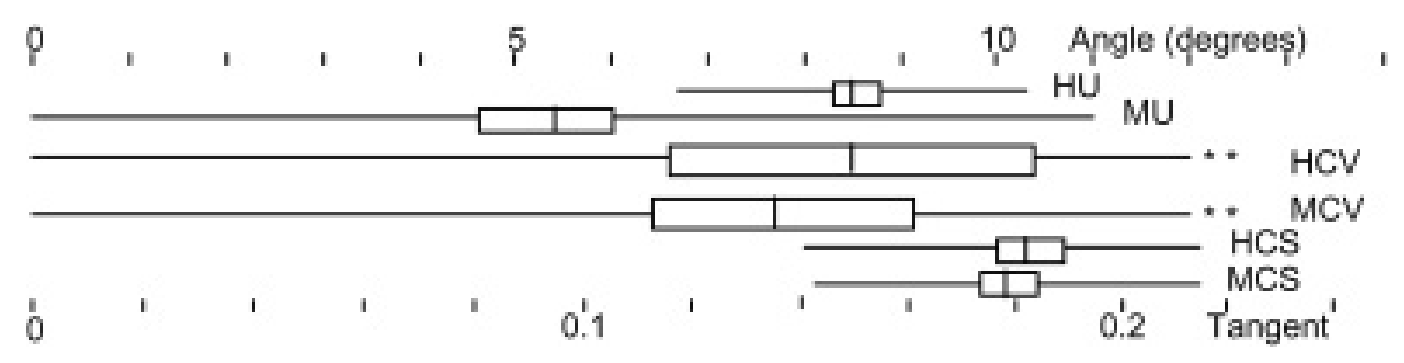

Fig. 6. Box and Whisker Plot: the slip angle from the Logistic Regression.

Table 2. Ranges of slip angle for different conditions

\begin{tabular}{lcccccc}
\hline Condition & Subject & Minimum & 1st quartile & 2nd Quartile & 3rd Quartile & Maximum \\
\hline Unconstrained & $\mathrm{H}$ & $6.7^{\circ} \backslash 0.12$ & $8.3^{\circ} \backslash 0.15$ & $8.5^{\circ} \backslash 0.15$ & $8.8^{\circ} \backslash 0.16$ & $10.3^{\circ} \backslash 0.18$ \\
& $\mathrm{M}$ & $0.0^{\circ} \backslash 0.00$ & $4.6^{\circ} \backslash 0.08$ & $5.4^{\circ} \backslash 0.09$ & $6.2^{\circ} \backslash 0.11$ & $11.0^{\circ} \backslash 0.19$ \\
Constrained Standing & $\mathrm{H}$ & $0.0^{\circ} \backslash 0.00$ & $6.6^{\circ} \backslash 0.12$ & $8.5^{\circ} \backslash 0.15$ & $10.4^{\circ} \backslash 0.18$ & - \\
& $\mathrm{M}$ & $0.0^{\circ} \backslash 0.00$ & $6.4^{\circ} \backslash 0.11$ & $7.7^{\circ} \backslash 0.14$ & $9.1^{\circ} \backslash 0.16$ & - \\
Constrained Seated & $\mathrm{H}$ & $8.0^{\circ} \backslash 0.14$ & $10^{\circ} \backslash 0.18$ & $10.3^{\circ} \backslash 0.18$ & $10.7^{\circ} \backslash 0.19$ & $12.1^{\circ} \backslash 0.21$ \\
& $\mathrm{M}$ & $8.1^{\circ} \backslash 0.14$ & $9.8^{\circ} \backslash 0.17$ & $10.1^{\circ} \backslash 0.18$ & $10.4^{\circ} \backslash 0.18$ & $12.1^{\circ} \backslash 0.21$ \\
\hline
\end{tabular}

For each subject and condition, angles corresponding to first, second (i.e., the median), and third quartiles were calculated, as were their associated coefficients of friction (the angle's tangent). The elements of the table show the angle in degrees $(x x)$ and the tangent (the friction coefficient, $y y)$ separated by a backslash $\left(x x^{\circ} \mid y y\right)$.

From the logistic regression equation, angles corresponding to first, second (i.e., the median), and third quartiles were calculated, as were their associated coefficients of friction (the angle's tangent). These results are presented in Table 2. The elements of the table show the angle in degrees and the tangent (the friction coefficient) separated by a backslash $\left(x x^{\circ} \backslash y y\right)$.

This is best visualized as a Box \& Whisker Plot (Fig. 6), where the vertical sides of the box with the line in it represent the first quartile (left side), the median (the line in the middle), and the third quartile (the right side of the box). The whisker represents the distance to the minimum (the left whisker) and the maximum (the right whisker. The asterisks denote the fact that the maximum is beyond the range of our analysis (but not by much, based upon our experience).

\section{Discussion}

All measurements are subject to measurement uncertainty. The probability function that quantifies that uncertainty is a measure of the quality of the measurement system. Measuring systems, expressed in the LogisticRegression framework, having a large enough slope $\left(\beta_{1}\right)$ would have a step-function-like response. Perhaps such results can be achieved when testing inert substances, but for biological, dynamic phenomena like walking and stepping, because there is a fair amount of inter-trial variability, such results are not generally achievable.
Then how does one test such in vivo systems? The answer is to impose a certain level of constraint (that which is achievable without compromising the system), and look at the probability functions as a measure of discriminatory capability. It is important to note that the increase in $\beta_{1}$ and convergence of the inter-subject logistic-regression functions as the constraint regime was tightened (from \{standing, unconstrained $\}$ to \{standing, constrained $\}$ to \{seated, constrained $\}$ ) reflects a real improvement in the mensuration capability of the system. This is (a) because the logistic-regression function is in fact the way to characterize a binary-output tribometers, such as the ramp tester used in these experiments (See Marpet and Medoff ${ }^{18,19)}$, Medoff et al. ${ }^{21,23)}$, Besser et al. ${ }^{20)}$, and Marpet et al. ${ }^{22)}$ ), (b) because foot hydration was controlled (See Naylor ${ }^{1}$, Sivamani et al..$^{8,10)}$, and Gitis and Sivamani ${ }^{9}$.), and (c) because the research clearly shows that, for a given anatomic skin area, no significant differences are found with respect to age, gender, and ethnicity $\left(\mathrm{Comaish}^{3)}\right.$, Cua et al..$^{4}$, and Egawa ${ }^{7}$.), implying that one would expect to see such convergence.

In evaluating the friction between the bare heel and wet marble, we used three methods: Unconstrained Standing, Constrained Standing, and Constrained Seated. For the Unconstrained Standing condition $(X \mathrm{U})$, variability (as described by the slope of the logistic regression) was fair to good, but the spread between subjects was wide, we hypothesize because of different stepping styles. For the Constrained, Standing Position (XCV), we found the sub- 
jects' characteristic curves significantly closer but, the discrimination of the system, as defined by the slope, $\beta_{1}$, fell by almost an order of magnitude.

Several contributors to the greater variability in the standing trials (both constrained and unconstrained) were recognized. The researchers noted step-to-step variation in heel strike location - as the subject lifted and dropped their foot on the test surface, at times it would land a little farther forward, at times farther back. While these strikes may only have been $4-6 \mathrm{~cm}$ apart, that small deviation would change the 'attack angle' of the foot to the test surface. This variability was even present in the constrained trials; while the leg was constrained by the carriage to travel vertically, the lower leg had to be allowed to rotate on the carriage, otherwise slip could not have occurred (the leg would have been held fast, even with no heel friction at all!). We suspect that some of the variability observed was due to this inconsistency in strike location. For the seated trials, the Operator would lift the carriage, the leg was allowed to hang vertically, the carriage was released and the foot allowed to drop to the test surface. Significantly more repeatability in heel strike location was observed.

Another possible cause of increased variability in the standing trials is active muscle control. For the standing subject to 'lower' their foot to the test surface, they were required to adduct the contralateral hip and/or flex the contralateral knee. Some reciprocal activation of hip musculature on the ipsilateral side would be expected, possibly changing the stiffness or inertial properties of the stepping leg, as well as heel strike location. As seated trials did not require muscle activity of the subject or the test leg, these trials were more repeatable.

There has been some discussion in the literature as to what actually constitutes a 'slip.' Leamon and $\mathrm{Li}^{25)}$ has described what he calls 'microslips' - slight movement between the foot and the floor that was 'too small' to be sensed by the walker, but could be observed visually. During our data collection, we did not consider microslips, and only looked for 'large' slips; however, as this distinction was made visually, it is probable that some inconsistency was introduced (It is difficult to decide how large a 'microslip' must become before it is considered a 'slip.')

Finally, visual observations of slip may be questioned overall as a reliable and valid method of slip detection. When observing a person walking in a laboratory, it is sometimes difficult to determine if they slipped. However, in our experimental protocol, we do not consider this relevant. In all trials, one researcher was solely focusing on slip detection, observing the foot-floor interface from a distance of approximately $30 \mathrm{~cm}$. At such a distance, and with no other distractions, we consider the detection of slip by visual observation to be accurate.

The Constrained Seated Position (XCS) offered the best performance: both low variability and low inter-subject spread, excellent intra-subject repeatability and excellent discrimination (a high value of $\beta_{1}$ ). We hypothesize that the improvement was caused by the leg motion being passive with respect to the subject. Because it eliminates much of the variability associated with active stepping by test subjects, it appears to offer the most promise as a standardized technique for in vivo characterization of barefoot pedestrian heel friction.

\section{Directions for Future Research}

In this paper, we have run ramp tests and utilized a mathematical characterization tool based upon Logistic Regression to determine if, in barefoot-human tribometric testing, the use of mechanical constraints on foot descent is justified. Clearly, it is. There are a number of approaches to extending this research.

First, a database of barefoot tribometric test results, with, among other variables, age, ethnic background, complexion, gender, foot condition, and hydration, would be useful to researchers and practitioners. Does barefootheel friction vary with respect to age? Skin complexion? Research on the forearm and other body parts indicates that there is no such variation. This needs to be checked with the heel. If such variation does exist, a cross-section of age $\times$ ethnic-background $\times$ complexion $\times$ etc. cohort could serve to assess within-cohort variability, so one could aggregate friction-similar subgroups.

The ramp that we used is not easily amenable to either biofidelity or to precision-and-bias assessments. That's because a force plate cannot (at least for our ramp) easily be incorporated into the ramp. Another significant problem is that pedestrians cannot walk on the ramp in anything resembling a normal manner, so that comparisons between the tribometer and gait-based metrics are simply not possible. (This is a criticism leveled against the German-developed DIN ramp tester, used for evaluation workplace floor surfaces.)

The design of this man-machine tribometer can be extended in a somewhat straightforward manner to a portable, field-testing unit. The authors are currently working on this device.

Finally, we intend to assess the biofidelity aspects of barefoot friction testing in two ways. First, we intend to replicate the test-surface and interface-contaminant conditions on an instrumented force plate, and have human subjects walk across that plate, with the conditions set so that at least some slips will occur. (At slip, it is the avail- 
able friction being measured, and not the required/utilized friction.) Secondly, and this is a long-term goal, we intend to install the portable tribometer above the force plate, to develop the characteristic-function-curve set of the tribometer, and see how the force plate mirrors those results. This will give insight into precision and bias of the field tester.

\section{References}

1) Naylor PFD (1955) The skin surface and friction. Br J Dermatol 67, 239-48.

2) Comaish S, Bottoms E (1971) The skin and friction: deviations from Ammontons' laws, and the effects of hydration and lubrication. Br J Dermatol 84, 37-43.

3) Comaish JS, Harborow PRH, Hofman DA (1973) A hand-held friction meter. Br J Dermatol 89, 33-5.

4) Cua AB, Wilhelm KP, Maibach HI (1990) Frictional properties of human skin: relation to age, sex and anatomical region, stratum corneum hydration and transepidermal water loss. Br J Dermatol 123, 473-9.

5) Loden M, Olsson H, Axell T, Linde YW (1992) Friction, capacitance, and transepidermal water loss (TEWL) in dry atopic and normal skin. Br J Dermatol 126, 137-41.

6) Asserin J, Zahouani H, Humbert Ph, Couturaud V, Mougin D (2000) Measurement of frication coefficient of the human skin in vivo: quantification of the cutaneous smoothness. Colloids Surf B Biointerfaces 19, $1-12$.

7) Egowa M, Oguri M, Hirao T, Takahashi M, Miyakawa M (2002) The evaluation of skin friction using a frictional feel analyzer. Skin Res Technol 8, 41-51.

8) Sivamani RK, Wu G, Gitis NV, Maibach HI (2003a) Tribological testing of skin products: gender, and ethnicity on the volar forearm. Skin Res Technol 9, 1-7.

9) Gitis NV, Sivamani R (2004) Tribometrology of skin. Tribology Transactions 47, 461-9.

10) Sivamani RK, Goodman J, Gitis NV, Maibach HI (2003b) Review-Coefficient of friction: tribological studies in man-an overview. Skin Res Technol 9, 227-34.

11) Li W, Qu SX, Zhou ZR (2006) Reciprocating sliding behavior of human skin in vivo at lower number of cycles. Tribology Letters 23, 165-9.

12) Brungraber R (1976) Evaluation of Slip-Resistance of Swimming Pool Deck Surfaces. Building Safety Section, Center for Building Technology, National Bureau of Standards, Maryland.

13) DIN 51097 (1992) Testing of floor coverings; determination of anti-slip properties; wet-loaded barefoot areas; walking method-ramp test, Deutsches Institut fur Normung, Berlin.
14) RAPRA procedure CH0001 (1997) Laboratory determination of the slip resistance of pedestrian flooring materials under water-wet conditions; walking-method ramp test. Rapra Technology, Ltd, UK.

15) Medoff HP, Brungraber R, Hilferty C, Mehta K (2002) Variable inclinable stepmeter: using test subjects to evaluate walkway surface/footwear combinations. Metrology of Pedestrian Locomotion and Slip Resistance, ASTM STP 1424, Marpet MI, and Sapienza MS (Eds.), ASTM International, Pennsylvania.

16) Hanson JO, Redfern MS, Mazumdar M (1999) Predicting slips and falls considering required and available friction. Ergonomics 42, 1619-33.

17) Burnfield JM, Powers CM (2004) Prediction of slip events during walking: An analysis of utilized coefficient of friction and available slip resistance. In: Proceedings of the American Academy of Forensic Sciences Meeting, Texas, 10, 145.

18) Marpet MI, Medoff HP (2004) The characterization of binary-output walkway-safety tribometric instrument by characteristic functions. In: Proceedings of the American Academy of Forensic Sciences Meeting, Texas, 10, 140-1.

19) Marpet MI, Medoff HP (2005) The characterization of binary-output walkway-safety tribometric instrument by characteristic functions: part 2. In: Proceedings of the American Academy of Forensic Sciences Meeting, New Orleans, 11, 143-4.

20) Besser M, Medoff HP, Marpet MI (2007) The effects of selected contaminants \& walking speed on tendency to slip as a function of foot velocity and acceleration. In: Proceedings of the 62nd STLE (Society of Tribologists and Lubrication Engineering) meeting, Philadelphia, on CD-ROM.

21) Medoff HP, Besser M, Marpet MI (2007a) The characterization of the Slip-Test Tribometer using logistic regression. In: Proceedings of the American Academy of Forensic Sciences Meeting, Texas, 12, 172.

22) Marpet MI, Medoff HP, Besser M (2007) Uncertainty Issues in tribometric testing: isolating the contribution of the tribometric instrument. In: Proceedings of the 62nd STLE meeting, Philadelphia, on CD-ROM.

23) Medoff HP, Marpet MI, Besser M (2007b) The characterization of binary-output tribometric instruments (Mark II and Mark III) by characteristic functions. In: Proceedings of the 62nd STLE meeting, Philadelphia, on CD-ROM.

24) (Example) Wikipedia, Logistic regression http://en.wikipedia.org/wiki/Logistic_regression. Accessed June 20, 2007.

25) Leamon TB, Li KW (1990) Microslip Length and the Perception of Slipping. In: Proceedings of the 23rd International Congress on Occupational Health, Montreal. 